\title{
Directed Chemical Assembly of Single and Clustered Nanoparticles with Silanized Templates
}

Calum Kinnear, ${ }^{\dagger}$ Jasper Cadusch, ${ }^{\ddagger}$ Heyou Zhang, ${ }^{\dagger}$ Jianing Lu, ${ }^{\dagger}$ Timothy D. James,,${ }^{\ddagger}$, Ann Roberts, $¥$ Paul Mulvaney ${ }^{\dagger}$

\author{
${ }^{\dagger} A R C$ Centre of Excellence in Exciton Science, School of Chemistry, University of \\ Melbourne, Parkville, Victoria 3010, Australia \\ ‡ School of Physics, University of Melbourne, Parkville, Victoria 3010, Australia \\ $\S$ Reserve Bank of Australia, Craigieburn, Victoria 3064, Australia
}

Correspondence to: calum.kinnear@unimelb.edu.au

\begin{abstract}
Assembly of nanoscale materials into arbitrary, organized structures remains a major challenge in nanotechnology. Herein, we report a general method for creating $2 \mathrm{D}$ structures by combining top-down lithography with bottom-up chemical assembly. Under optimal conditions, assembly of gold nanoparticles was achieved in less than 30 minutes. Single gold nanoparticles, from 10 to $100 \mathrm{~nm}$, can be placed in predetermined patterns with high fidelity, and higher order structures can be generated consisting of dimers or trimers. It is shown that the nanoparticle arrays can be transferred to, and embedded within, polymer films. This provides a new method for the large-scale fabrication of nanoparticle arrays onto diverse substrates using wet chemistry.
\end{abstract}




\section{Introduction}

Adsorbing solution-dispersed nanoparticles onto solid surfaces in specific patterns holds great promise for the generation of functional materials and devices. ${ }^{1-2}$ However, nanoparticle motion is dominated by thermal fluctuations, which renders controlled deposition at surfaces extremely challenging. While there have been remarkable advances in bottom-up self-assembly using molecules such as DNA as templates, ${ }^{3-5}$ these methods cannot be easily translated to the assembly of spatially well-defined structures on 2D surfaces. To accomplish this, top-down lithography tools are normally utilized. These tools have advanced to the point where it is now possible to generate sub-20 nm features on surfaces in a scalable and reproducible fashion, ${ }^{6}$ enabling the assembly of nanoparticles to form novel plasmonic surfaces, ${ }^{7}$ sensing substrates, ${ }^{1}$ and photovoltaics. ${ }^{8}$ However, while the lithographic fabrication of gold nanostructures via direct evaporative processes appears simpler, solution-synthesized single crystal metal nanoparticles exhibit reduced surface plasmon damping and superior optical properties. ${ }^{9-10}$ Additionally, it is not possible to lithographically fabricate more complex materials such as cubes or core-shell structures. Therefore, methods to assemble complex nanoparticles directly from suspension remains an outstanding scientific challenge.

To be adopted within the field of advanced printing technologies, an assembly method must (a) work for single nanoscale objects, (b) yield nanometer spatial resolution, (c) be compatible with a wide range of particles, (d) be scalable, and (e) work for different substrates.

One common approach has used-been to employ nanolithography. Lithographic tools have been utilized in several ways to direct the assembly of nanoparticles, via physical or electrostatic/chemical interactions with patterned substrates. Drawing a colloidal suspension over a solid patterned substrate can result in the deposition of nanoparticles into the etched pattern due to capillary and convective forces at the receding contact line (CFA). ${ }^{11-17}$ This has been successfully applied to the assembly of a wide variety of materials, shapes, and sizes, ranging from inorganic nanoparticles to viruses. ${ }^{18-19}$ While CFA places no specific constraints on the nanoparticle material or surface charge, it 
requires rigorous control over multiple variables, e.g. humidity, temperature, interfacial contact angle, particle volume fraction, and substrate velocity. ${ }^{16}$ Additionally, any pinning of the receding meniscus induced by surface roughness results in deposition of large rafts of particles randomly on the substrate. ${ }^{20}$ Hence, CFA is very difficult to scale $\mathrm{up}_{2}$ although for small scale assembly it is a very practical method. An alternative, serial approach is optical printing with focused laser light leading to deposition of metallic nanoparticles with high accuracy, dependent on the overlap between wavelength of the laser and the localized surface plasmon resonance of the nanoparticle. ${ }^{21-22}$ This appears very promising for plasmonic materials but, being a serial method that requires $>1$ s per nanoparticle, the potential for high throughput assembly of with this technology is currently limited.

Electrostatic charges patterned onto surfaces in a xerography-like approach can also be used to direct the assembly of charged nanoparticles. ${ }^{23-25}$ This is normally accomplished in a serial manner by selective charging of the substrate with an atomic force microscopy (AFM) tip. Alternatively, (di)electrophoresis and electrical nanoimprint lithography both have the potential to achieve parallel electrostatic assembly of nanomaterials, but scalable fabrication remains elusive. ${ }^{26-27}$

A second important aspect of any nanoscale chemical assembly process is the ability to employ different substrates. In some cases, transfer of printed structures is possible. For example, in the case of directed chemical assembly (DCA), the development of silicones as $\mu$-contact printing elements has enabled stamping of molecules in predetermined patterns onto surfaces, e.g. self-assembled monolayers of aminothiols can be stamped onto gold surfaces. ${ }^{28-30}$ These can then be used to direct the assembly of nanoparticles in a parallel manner via either electrostatically attracting nanoparticles to the patterns or adsorbing them through shorter range chemical interactions upon diffusion to the surface. ${ }^{30}$ This has been widely used to assemble carbon nanotubes and graphite oxides due to their strong interaction with $\mathrm{COOH}$ terminated surfaces. ${ }^{31-32}$ Combining electrostatically repulsive backgrounds with attractive templates can also 
achieve the assembly of sub-100 nm nanoparticles with high spatial resolution. ${ }^{33}$ While parallel, and rapid, significant obstacles remain for $\mu$-contact printing enabled DCA. The spatial resolution, in roll-to-roll systems, is currently limited to around $300 \mathrm{~nm},{ }^{34}$ which is surpassed by nanoimprint lithography tools, ${ }^{35}$ and ink spreading is a problem for high resolution nanostructures. ${ }^{36}$ Additionally, the majority of studies presenting high quality $\mu$-contact printing have done so with self-assembled monolayers of thiols on gold surfaces, limiting the choice of substrate.

Single nanoparticles can also be assembled, via DCA, onto films of block copolymers where one block strongly interacts with the nanoparticle while the other repels it. This has been used to assemble rows of aligned gold nanorods, hexagonally packed gold nanospheres, or lines of clustered gold nanospheres. ${ }^{37-40}$ These assembly methods are extremely attractive due to their scalability and rapid parallel assembly $y_{2 ;}$, however, forming the formation of arbitrary patterns cannot be achieved utilizing this bottom-up method. For this, a combined DCA method that uses both top-down and button-up techniques is needed.

DCA approaches, which avoid the use of $\mu$-contact stamps, have been demonstrated previously where molecules are absorbed from solution, rather than stamped, onto substrates which are patterned via other lithographic means. Gilles et al. described a method where silicon templates were patterned via nanoimprint lithography followed by silanization of the exposed substrate with amino-groups and resist lift-off. ${ }^{41}$ Citrate coated gold nanoparticles were then adsorbed to the patterns in clusters or as single nanoparticles with reasonable defect control. Reinhard and coworkers have also generated clusters of gold nanoparticles in periodic arrays with patterned poly-lysine on a PMMA background as a selective binding group. ${ }^{42}$ However, clusters were assembled over 12 hours and a lift-off step was necessary to remove non-specifically bound particles. Chi and coworkers have also used a DCA method to assemble gold nanoparticles over 8-12 hours..$^{43-44}$ They functionalized a substrate with amine groups, before coating with PMMA to enable the writing of a pattern with electron-beam lithography (EBL). The gold nanoparticles adsorbed to the amine groups present at the bottom of each cavity. This approach is incompatible with the more scalable nanoimprint 
lithography tools, where a pattern transfer step via reactive ion etching is needed to clean the bottom of each cavity to expose the substrate. ${ }^{45}$

Of the above studies None of the above studies, none could rapidlycould demonstrate rapid assemble assembly of single nanoparticles, in the range of 1 10-100 $\mathrm{nm}$ in diameter), over large $\left(>1 \mathrm{~cm}^{2}\right)$ areas $\left(>1 \mathrm{~cm}^{2}\right)$ - _utilizing methods that are compatible with proven scalable fabrication tools. Here, we show that a modified form of DCA that combines simple dip-coating wet-chemistry with nanoimprint-compatible electron beam lithography, can achieve high fidelity patterns of single or clustered gold nanoparticles in a rapid and parallel manner. We employ gold nanoparticles for proof of principle, but the method should be extendable to other colloidal systems. ${ }^{46-47}$ We demonstrate that single nanoparticles down to $10 \mathrm{~nm}$ in diameter can be deposited with nanometer spatial control. The approach is comprised of two basic steps. Firstly, electron beam lithography is used to fabricate nanometer sized wells in PMMA substrates. To localize single nanoparticles in each well, we chemically modify the bottom of the wells with molecular adhesives that bind any nanoparticles that diffuse into the well. The approach is scalable and can be applied to a wide range of particle compositions, shapes and sizes.

\section{Experimental Section}

All chemicals were ACS reagent grade, obtained from Sigma-Aldrich, and used as supplied unless otherwise specified. Cetyltrimethylammonium bromide (CTAB) and sodium oleate were supplied by $\mathrm{TCl}$, and sodium citrate was from Chem-Supply. Silicon and glass wafers were obtained from University Wafer. All glassware used to synthesize nanoparticles was cleaned in freshly prepared aqua regia before extensive rinsing with Milli-Q water.

Synthesis of nanoparticles. Gold nanospheres were synthesized via a kineticallycontrolled seeded growth method. ${ }^{48}$ Briefly, an aqueous solution of sodium citrate (150 $\mathrm{mL}, 2.2 \mathrm{mM}$ ) was refluxed in a round bottomed flask for 10 min under continuous stirring before adding extra sodium citrate $(2 \mathrm{~mL}, 60 \mathrm{mM})$ immediately followed by $\mathrm{HAuCl}_{4}$ (0.555 mL, $45 \mathrm{mM})$. After approximately 6-7 mins the solution turned a light red color. 
Boiling was continued for a further $10 \mathrm{~min}$ before reducing the temperature to $85^{\circ} \mathrm{C}$. Two more additions of $\mathrm{HAuCl}_{4}(0.555 \mathrm{~mL}, 45 \mathrm{mM})$ separated by 20 min were then made. An aliquot ( $55 \mathrm{~mL}$ ) was removed from the reaction flask, followed by the addition of Milli-Q water preheated to $60{ }^{\circ} \mathrm{C}(52.3 \mathrm{~mL})$. Once the temperature of the reaction returned to $85{ }^{\circ} \mathrm{C}$, sodium citrate was added $(1 \mathrm{~mL}, 60 \mathrm{mM})$ followed by three additions of $\mathrm{HAuCl}_{4}(0.555 \mathrm{~mL}, 45 \mathrm{mM})$ again separated by $20 \mathrm{~min}$. The dilution and growth steps were then repeated 10-12 times, with adjustments in the volume of sodium citrate (1-2 $\mathrm{mL}$ ) to maintain $\mathrm{pH}$ 6-7.

Gold nanorods were synthesized following a modified mixed surfactant route using sodium oleate and CTAB. ${ }^{49}$ Briefly, seed nanoparticles were synthesized by mixing $\mathrm{HAuCl}_{4}(5 \mathrm{~mL}, 0.5 \mathrm{mM})$ and CTAB $(5 \mathrm{~mL}, 0.2 \mathrm{M})$ followed by $\mathrm{NaBH}_{4}(0.3 \mathrm{~mL}, 20 \mathrm{mM})$ under vigorous stirring. A growth solution was then prepared containing CTAB $(9 \mathrm{~g})$, sodium oleate $(1.543 \mathrm{~g}), \mathrm{AgNO}_{3}(18 \mathrm{~mL}, 4 \mathrm{mM}), \mathrm{HAuCl}_{4}(4.739 \mathrm{~mL}, 51.9 \mathrm{mM}), \mathrm{HCl}(2.5$ $\mathrm{mL}, 32 \%)$, L-ascorbic acid $(1.25 \mathrm{~mL}, 0.1 \mathrm{M})$, and seeds $(0.4 \mathrm{~mL})$ aged for $2 \mathrm{~h}$.

Silver nanospheres were synthesized via a similar kinetically-controlled seeded growth as the gold nanospheres. ${ }^{50}$

Template preparation. Silicon or glass wafers $(10 \mathrm{~cm})$ were hydroxylated in piranha solution over $1 \mathrm{~h}$ before extensive rinsing with Milli-Q water, acetone, and isopropyl alcohol (IPA). The wafer was briefly dehydrated on a hot plate at $180^{\circ} \mathrm{C}$ before spincoating PMMA (950PMMA, A2, MicroChem) at 3,000 rpm and baking on a hot plate at $170{ }^{\circ} \mathrm{C}$ for $5 \mathrm{~min}$. A thin layer of chromium was then evaporated onto the PMMA coated glass wafer using an Intlvac Nanochrome II. The wafers were then exposed on a Vistec EBPG5000plusES and the chromium removed with Transene $\mathrm{Cr}$ etchant for 25 seconds followed by $5 \% \mathrm{H}_{2} \mathrm{SO}_{4}$ for 20 seconds. Pattern development was in 1:3 MIBK:IPA for 1 min followed by an IPA rinse.

Assembly. The silanization of a developed template was carried out in a solution containing ethanol (95\%), Milli-Q water $(3 \%)$, acetic acid $(1 \%)$, and silane $(1 \%, \mathrm{~N}-(3-$ (trimethoxysilyl)propyl)ethylenediamine). The silane was added to the acidic ethanol and allowed to hydrolyze for $10 \mathrm{~min}$ before submersion of the template in the solution for 1-2 min. If the concentration of water increases above $3 \%$ then further development 
of the PMMA can damage the pattern therefore absolute ethanol is used in the silanization solution.

The gold and silver nanospheres were functionalized with either bis(psulfonatophenyl)phenylphosphine or tris(2-carboxyethyl)phosphine (2 mM) overnight, followed by addition of sodium chloride to obtain the desired ionic strength.

Gold nanorods were functionalized with polyvinylpyrrolidone $(1 \mathrm{mM})$ followed by transfer to acidified methanol. Mercaptoundecanoic acid was then added and left to react over $48 \mathrm{~h}$ with purification by three rounds of centrifugation into Milli- $\mathrm{Q}$ water.

Gold nanosphere assembly was induced by dip-coating the silanized template in the suspension for various times between $30 \mathrm{~s}$ and $30 \mathrm{~min}$., depending on the well density. Gold nanorod assembly necessitated a $24 \mathrm{~h}$ incubation in the suspension with the template.

Array transfer. The arrays were transferred into PDMS through the curing of Sylgard $(\mathbb{R}$ 184 on the substrate followed by lift-off. More specifically, after assembly the arrays were extensively rinsed with Milli-Q water followed by a plasma etch to decrease the thickness of the PMMA to 1-5 nm and left overnight at RT. Freshly prepared PDMS was degassed under vacuum before casting over the array and curing for $4 \mathrm{~h}$ at $70{ }^{\circ} \mathrm{C}$. The cured film was then carefully peeled off the substrate and transferred to a glass slide for characterization by AFM and light microscopy. A similar method was used to transfer the array into a PVA film through casting of a degassed aqueous PVA solution over the arrays and allowing the water to evaporate over $24 \mathrm{~h}$ at RT (10 mg.mL $\mathrm{mL}^{-1}, 88 \%$ hydrolyzed). The thin film could then be carefully peeled off the substrate and transferred to a glass slide for characterization.

\section{Results}

Our approach to chemically direct the assembly of single nanoparticles is summarized in Scheme 1. Wells of varying sizes are etched into a thin film of PMMA (typically $50 \mathrm{~nm}$ in depth), on either silicon or glass, by standard EBL methods. The base of the wells is then cleaned of residual polymer via brief plasma cleaning under ozone. The hydroxyl 
groups on the silicon dioxide surface at the bottom of these wells can then be selectively functionalized to create patches, which specifically bind nanoparticles from suspension. The adhesion can be electrostatic; for example, citrate capped gold particles are negatively charged so a cationic surface could be employed.
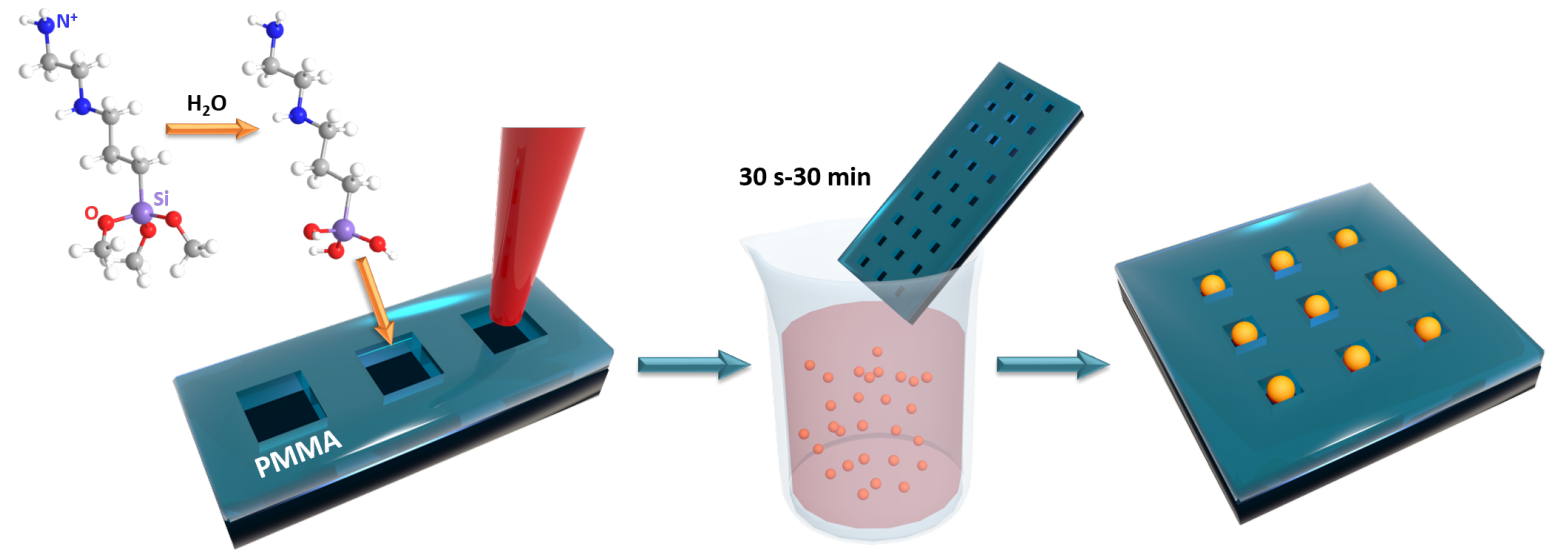

Scheme 1. (a) Wells are etched into a $50 \mathrm{~nm}$ thick PMMA layer using electron beam lithography followed by aqueous phase silanization of the underlying hydroxylated substrate. While gold particles do not adsorb to PMMA, the amine groups can chemically bind them. The amine groups carry a positive charge below $\mathrm{pH}$ 9. (b) The substrate is dipped into a suspension of negatively charged nanoparticles. (c) Well filling occurs via diffusion and leads to their assembly in the wells as either single particles (as shown here), or as clusters depending on the ratio of well size to nanoparticle size.

Alternatively, more sophisticated molecular adhesive approaches can be employed such as: click chemistry, DNA based recognition, thiol-alkene chemistry or streptavidinbiotin linkers. Here we have utilized the fact that amine moieties bind strongly to gold surfaces displacing citrate or phosphine groups, aided by the wide variety of amine terminated silanes that are readily available. As highlighted in Scheme 1, treatment with silanes such as aminopropyl trimethoxysilane leads to a sharp chemical contrast between the top of the PMMA and the underlying substrate. While alternative routes, such as selective poly-lysine coatings ${ }^{42}$ or functionalization prior to applying the resist, ${ }^{51}$ may give a similar result, we found them to result in high levels of non-specific 
adsorption. Such pre-patterning is also incompatible with nanoimprint lithography, which normally requires a plasma-etch pattern transfer step. This step removes the underlying molecular adhesive layer. ${ }^{42-43,51}$ Conversely, our approach here can be used without PMMA lift-off. Once the surface has been prepared, the glass substrate may be simply dip-coated into the nanoparticle suspension. The PMMA surface is hydrophobic and the tendency for adsorption of gold particles to the PMMA is low. To improve the quality of the gold nanoparticle arrays, the effect of several parameters including the nanoparticle concentration, $\mathrm{pH}$, ionic strength, dip-coating period, the well chemistry, and the well geometry were investigated.

Well Chemistry. We have found that the optimum assembly is obtained with (i) polyamine based silanes rather than aminopropyl triethoxysilane treatment, and (ii) a conventional, aqueous hydrolysis-condensation silanization reaction (Supporting Information, Figure S1). Thiolated templates, without attractive electrostatic particlesubstrate interactions, resulted in very low quantities of deposited nanoparticles. Conversely, cationic quaternary ammonium silanes resulted in many deposited nanoparticles, despite the lack of donating electron density.

Dip-coating of the amine-functionalized templates into a suspension of oppositely charged nanoparticles results in their assembly into the wells over a matter of minutes (Scheme 1), orders of magnitude faster than the 8-24 $\mathrm{h}$ previously required. $42,44,51$ While longer times can be used to increase the filling ratios, non-specific adsorption of nanoparticles to the background PMMA increases, thus necessitating resist lift-off, which is only required if a pattern of high fidelity is required.

Well Dimension and lonic Strength. A key question is how to determine the optimal well size given a certain particle size. Clearly it needs to be wide enough to accommodate the adsorbing particle but if it is too large, then dimers may also infiltrate the well. At the same time, a larger well increases the rate of filling. The challenge is to maximize the filling rate while maintaining single nanoparticle occupancy within each well.

In Figure 1a we present the percentage of filled wells and number of particles per well for $46 \mathrm{~nm}$ and $117 \mathrm{~nm}$ diameter gold particles, as a function of the square well size. The 
nanoparticles are bis(p-sulfonatophenyl)phenylphosphine (BSPP) capped with a zeta potential of $-41 \mathrm{mV}$, and concentrations of $300 \mathrm{pM}$ and $21 \mathrm{pM}$ respectively. The ionic strength is maintained at $2 \mathrm{mM}$ with phosphate buffer, and a $\mathrm{pH}$ of 7.4 to ensure the amine groups of the silanes are mostly protonated. The templates were functionalized with $\mathrm{N}$-(3-(trimethoxysilyl)propyl)ethylenediamine. The occupancy was measured from SEM images of 50 identical wells spaced $6 \mu \mathrm{m}$ apart over $300 \mu \mathrm{m}$ in triplicate. The well dimensions were measured by SEM, with an associated random uncertainty of $4 \%$ originating from the resolution of the SEM and the image analysis routine. The measurements were consistent across arrays spaced $1 \mathrm{~cm}$ apart. Notably, we see that for the $46 \mathrm{~nm}$ particles, there is only 1 particle per well, even up to well sizes of $140 \mathrm{~nm}$, which could potentially accommodate 3 particles in a row. Above $140 \mathrm{~nm}$, we begin to observe double occupancy. For the $117 \mathrm{~nm}$ nanoparticles, the situation is similar, except the effects are displaced to larger well sizes. Single occupancy is observed up to $200 \mathrm{~nm}$ well sizes and then multiple occupancy becomes more likely. For both sizes, there is an overlapping window where one observes single occupancy with low vacancy: for $46 \mathrm{~nm}$ nanoparticles this is $160-180 \mathrm{~nm}$, and for $117 \mathrm{~nm}$ nanoparticles this is between $200-220 \mathrm{~nm}$ for an ionic strength of $2 \mathrm{mM}$.

In Figure 1b, an SEM micrograph is presented showing the clean deposition of single 13 $\mathrm{nm}$ particles into the wells - a scale that is extremely challenging to reach with evaporative methods. Note that the change in concentration of suspended particles due to adsorption is negligible and is effectively constant throughout the process.

Upon increasing the ionic strength of the colloidal suspension, we observed more nanoparticles depositing into each well. This is shown in Figure 1c, where we vary the ionic strength. Here, we observe the occupancy of $72 \mathrm{~nm}$ gold particles in wells of different widths (the well depth is fixed at $50 \mathrm{~nm}$ ), with $\mathrm{pH}$ fixed at 7.4 and the gold nanoparticle concentration fixed at $78 \mathrm{pM}$. The occupancy is found to depend strongly on the ionic strength, where increasing salt concentrations leads to higher occupancy with very low vacancy values.

In Figure 1d we see the results of this surface coagulation in an SEM image, which shows multiple $72 \mathrm{~nm}$ particles condensing into a $230 \mathrm{~nm}$ wide well due to an ionic 
strength of $24.5 \mathrm{mM}$. Adding salt did not increase the non-specific adsorption to the PMMA, which remains relatively unaffected. This is effectively a type of ordered surface coagulation. Figure 2 shows higher magnification examples of this where $72 \mathrm{~nm}$ gold spheres were assembled into a variety of trimers, hexamers, and other kinetically trapped structures within $250 \mathrm{~nm}$ wide wells at high ionic strength.

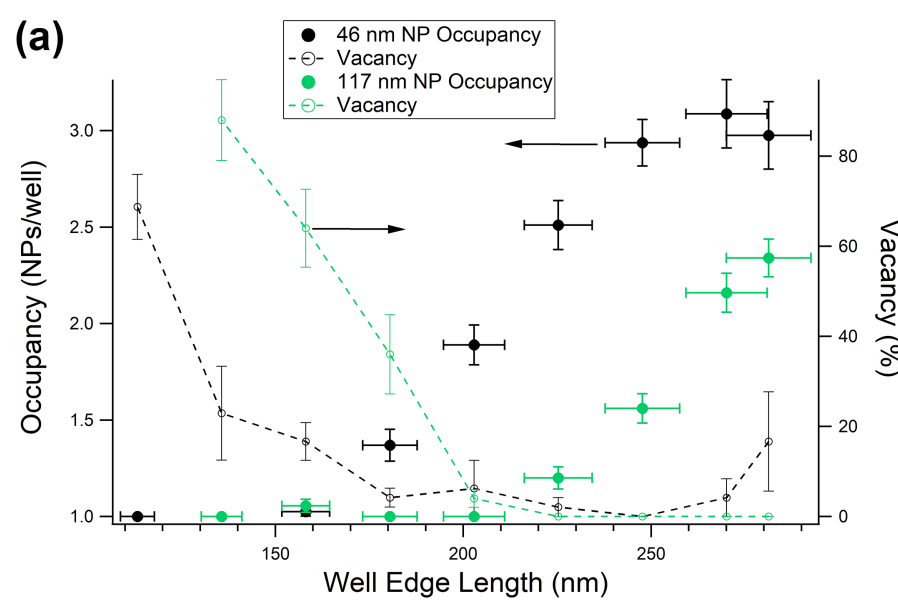

(b)
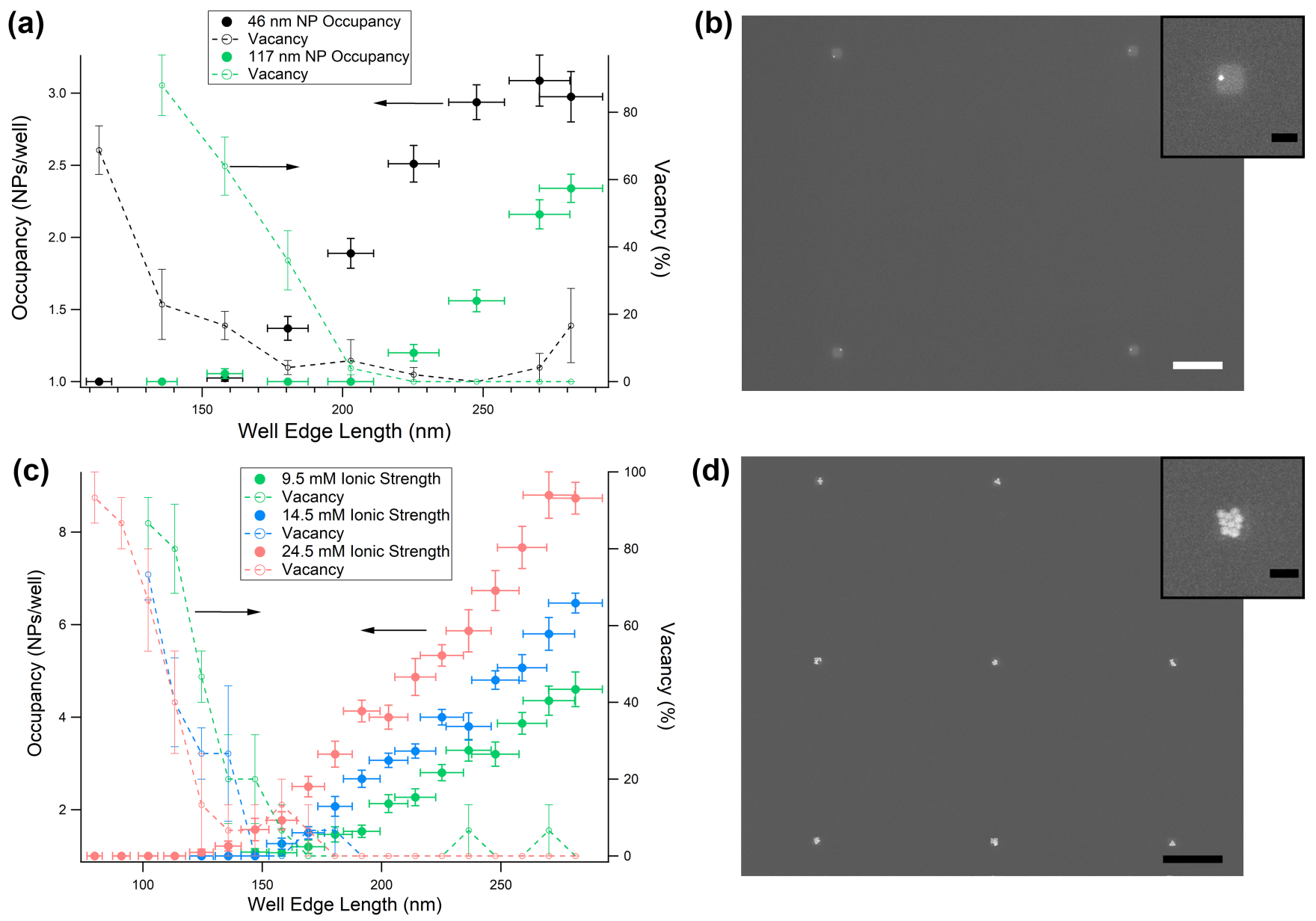

(d)

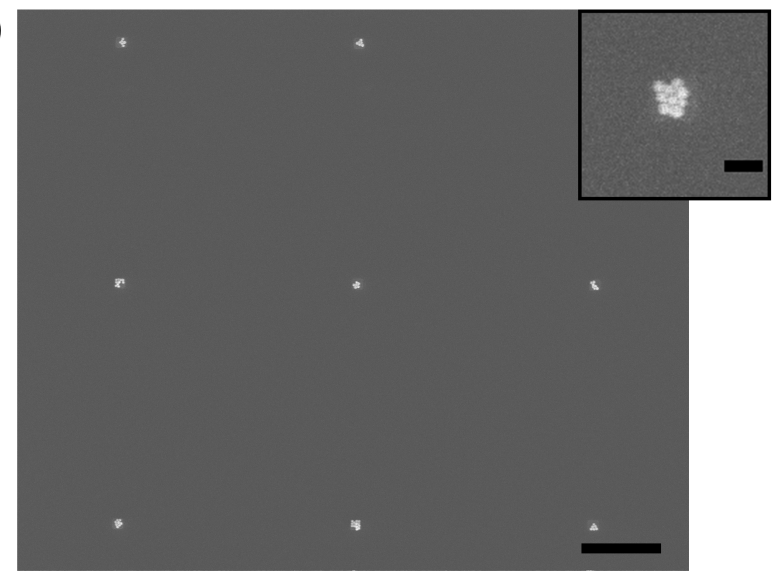

Figure 1. (a) The observed occupancy, defined as the number of particles per well, and the vacancy, defined as the percentage of empty wells, of square wells as a function of the well dimension and particle size for 46 and $117 \mathrm{~nm}$ gold nanospheres in $2 \mathrm{mM}$ phosphate buffer at 21 ${ }^{\circ} \mathrm{C}$ after incubation for $30 \mathrm{~min}$ in a suspension with concentrations of $0.3 \mathrm{nM}$ and $21 \mathrm{pM}$ respectively. The PMMA depth is $50 \mathrm{~nm}$, solution $\mathrm{pH}=7.4$, ionic strength is $2 \mathrm{mM}$, the templates were functionalized with N-(3-(trimethoxysilyl)propyl)ethylenediamine. (b) SEM micrograph of $13 \mathrm{~nm}$ gold nanoparticles deposited in wells with an edge length of $150 \mathrm{~nm}$ without any PMMA lift-off steps. Scale bar, $1 \mu \mathrm{m}$ and $150 \mathrm{~nm}$ (inset). (c) Filling efficiency as a function of the ionic strength of a $72 \mathrm{~nm}$ gold nanoparticle suspension. Ionic strength was varied by the addition of $\mathrm{NaCl}, \mathrm{pH}=7.4$, and the nanoparticle zeta potential was $-41 \mathrm{mV}$. (d) SEM micrograph of $72 \mathrm{~nm}$ 
nanoparticles in $230 \mathrm{~nm}$ wells assembled at $24.5 \mathrm{mM}$ ionic strength, as in (c). Scale bar, $2 \mu \mathrm{m}$ and $230 \mathrm{~nm}$ (inset).
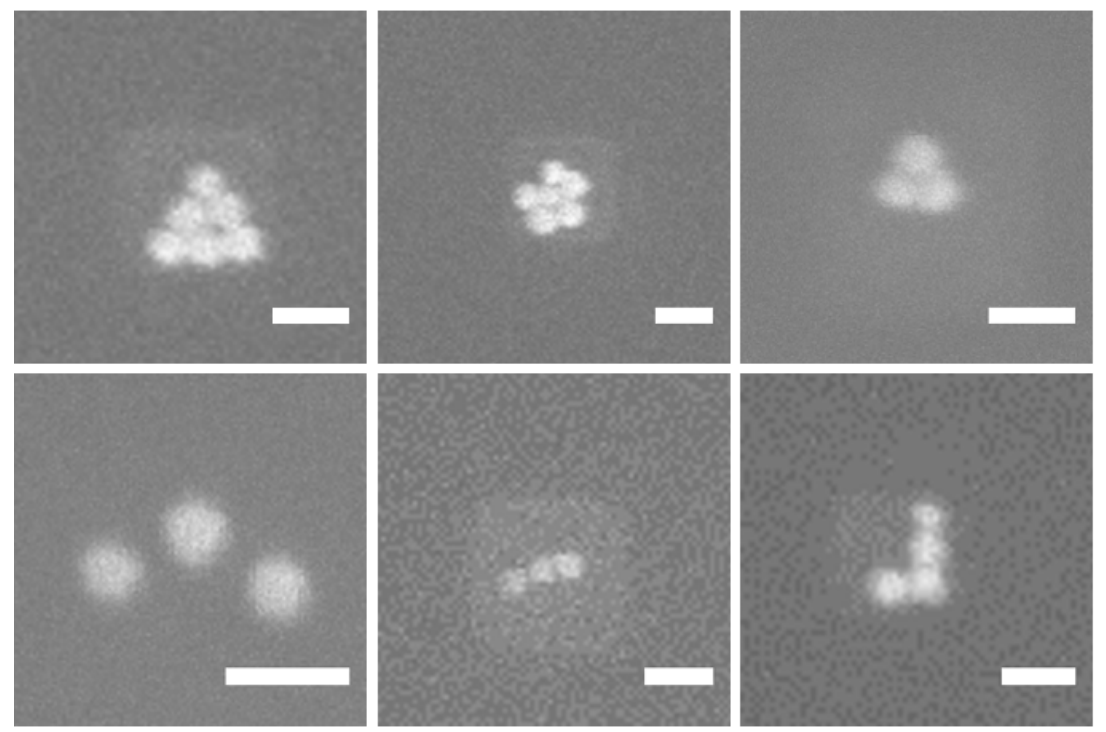

Figure 2. SEM micrograph of $72 \mathrm{~nm}$ gold nanoparticles assembled at $24.5 \mathrm{mM}$ ionic strength, as described and shown in Figure 1c, into $250 \mathrm{~nm}$ wide wells etched in a $50 \mathrm{~nm}$ thick PMMA film on silicon. Scale bar, $150 \mathrm{~nm}$.

Well Filling Kinetics. Figure 3a presents the percentage of empty wells upon incubation with BSPP-capped $72 \mathrm{~nm}$ gold nanoparticles in $150 \mathrm{~nm}$ wide wells at a fixed ionic strength of $9.5 \mathrm{mM}, \mathrm{pH} \mathrm{7.4}$, at three times: $30 \mathrm{~s}, 3 \mathrm{~min}$, and $30 \mathrm{~min}$. For each time point and well spacing, the occupancy of 360 wells were determined over 4 repeats. After a $30 \mathrm{~min}$ incubation, all wells spaced farther apart than $300 \mathrm{~nm}$ were filled, irrespective of ionic strength, as shown in Figure $3 b$ where the experiment was replicated once more at four different ionic strengths. When the separation was less than $200 \mathrm{~nm}$, we observed greater clustering and non-specific adsorption to the interwell PMMA background. At this separation, the density of hydrophilic areas to hydrophobic background is too high to form a smooth contact line. Notably, when the substrate was removed from the suspension, meniscus pinning can also occur on highly hydrophilic areas, as observed during capillary force assembly, leading to non-specific adsorption. ${ }^{20}$ 
In Figure 3c, we have expressed the data in Figure $3 a$ as the percentage of wells filled (100\%-vacancy) for each spacing against time. After 3 min the wells furthest apart are filled fastest. These data were fitted by a power law, which are shown as dashed lines in Figure 3c.

In an ideal situation where there is only a hard-sphere repulsive interaction between nanoparticles, the number of nanoparticles reaching and sticking to a finite macroscopic surface due to diffusion decays as $t^{1 / 2}$, therefore we expect the exponent of the power fit to be close to 0.5 in Figure 3c. In Figure $3 \mathrm{~d}$ the exponents extracted from the fits of Figure $3 c$ are plotted as a function of well-spacing - note we are not fitting a function here, simply comparing the fitted exponent of a power fit with what would be expected from purely diffusion-limited assembly. The furthest apart wells, which can be considered non-interacting, indeed have an exponent close to the theoretical value of 0.5 , consistent with diffusion-limited filling. As the spacing is decreased, the exponent increases, potentially reflecting a local increase in the gold particle concentration. For wells separated by distances of 400 to $600 \mathrm{~nm}$, near complete deposition was found after 30 min leading to arrays of different colors due to diffractive effects (Figure S2). 
(a)

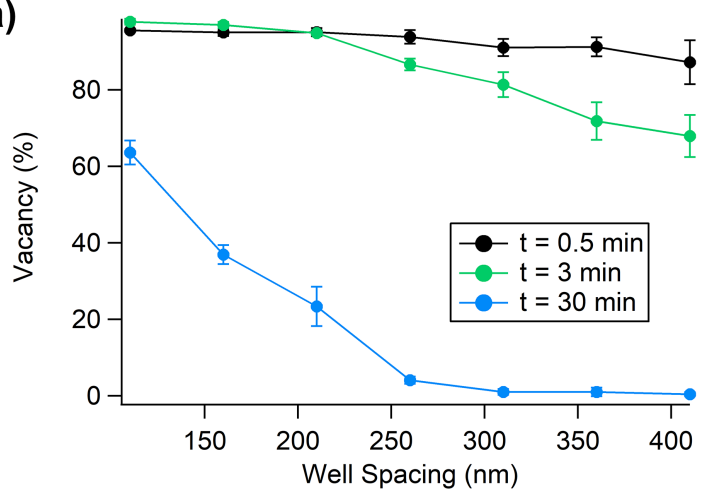

(c)

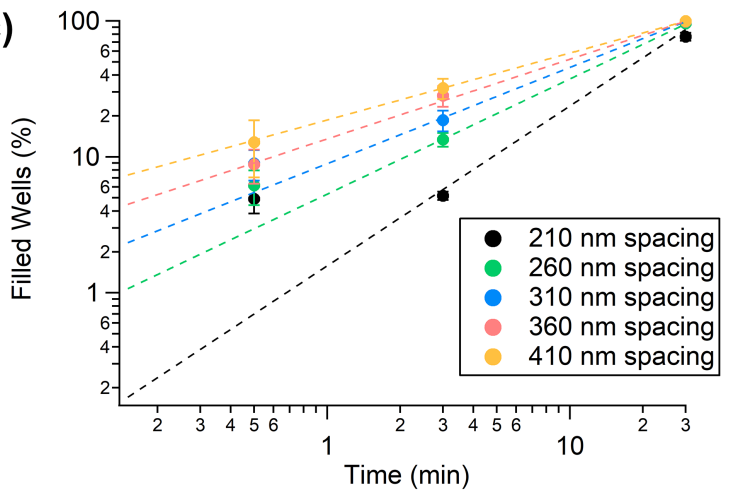

(b)

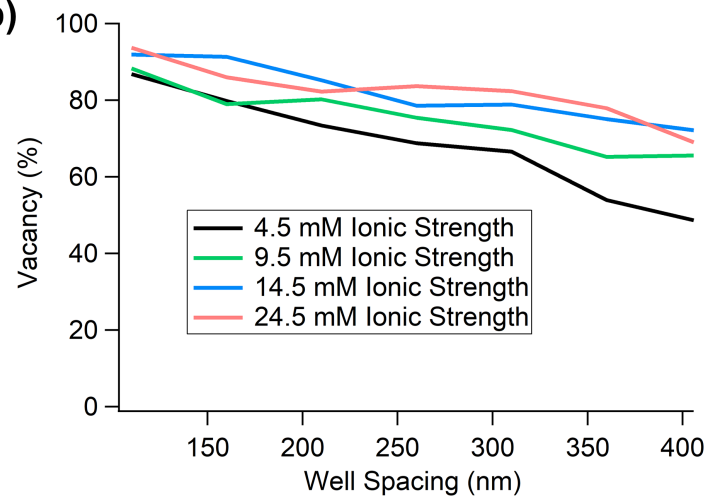

(d)

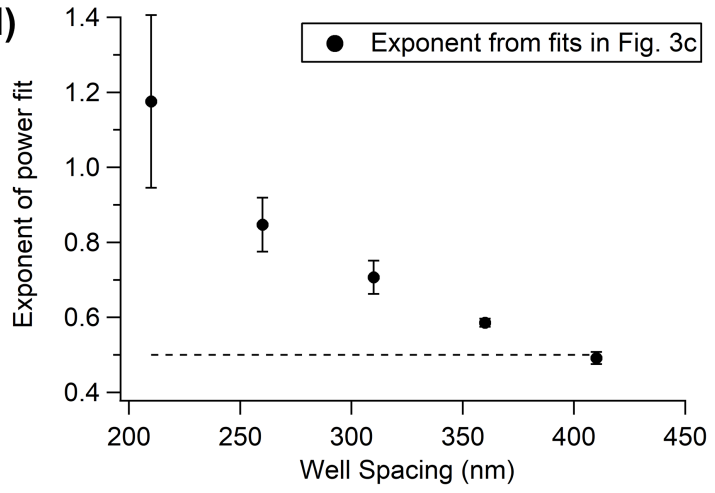

Figure 3. (a) Percentage of unfilled wells (vacancy) as a function of time and well spacing, defined as the edge-to-edge distance between wells for $72 \mathrm{~nm}$ gold nanoparticles functionalized with BSPP at 78 pM, pH 7.4, and $9.5 \mathrm{mM}$ ionic strength. Error bars are standard deviation over 4 measurements of 360 wells per experimental condition. (b) Well vacancy as a function of ionic strength after $3 \mathrm{~min}$, where the conditions were the same as in (a) and the ionic strength was varied by the addition of $\mathrm{NaCl}$. (c) Power-law fit to the percentage of filled wells from (a), as a function of time for various well spacings. Dashed lines correspond to the fits and error bars are standard deviation over 4 measurements of 360 wells per experimental condition. (d) Exponents of the power-law fits from (c) as a function of the well spacing. Error bars are standard error from the fit.

Nanoparticle Surface Chemistry. In addition to tuning the substrate surface chemistry, the stabilizing ligands present on the nanoparticle surface play a major role in minimizing any non-specific binding to the PMMA mask - ideally $99.999 \%$ of the surface is nanoparticle-free after any assembly procedure. $\$$ The importance of capping ligands can be seen in Figure S3 where arrays with a separation of $1 \mu \mathrm{m}$ were assembled over $30 \mathrm{~min}$ at the same $\mathrm{pH}$, buffer concentration, ionic strength, and nanoparticle concentration as used in the experiments in Figure 1a, except that four different capping

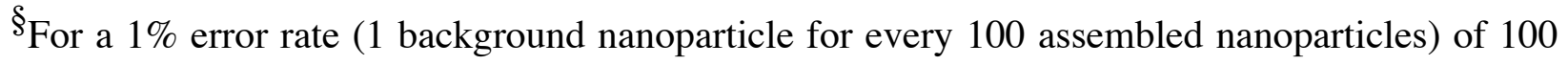
$\mathrm{nm}$ diameter nanoparticles on an array of $5 \mu \mathrm{m}$ interparticle spacing. 
ligands were used: tris(2-carboxyethyl)phosphine (TCEP), BSPP, mercaptoundecanoic acid (MUA), and sodium citrate. Nanoparticles assembled into the wells when the two phosphine ligands were used; however, very little assembly occurred for the MUA case. Sodium citrate capped nanoparticles also adsorbed non-specifically to the background as well as in the arrays.

Gold nanorods and silver nanospheres. While spherical nanoparticles are commonly deposited into arrays, other geometries such as rods are appealing due to their polarization dependent optical properties as well as other physicochemical properties. However, the surface chemistry of gold nanorods is more complex than that of spheres due to the presence of strongly bound CTAB meaning ligand exchange is not straightforward. ${ }^{52-53}$ To address this issue, we developed a route to negatively charged gold nanorods without the use of thiolated polymers such as mercapto-PEG. Firstly, polyvinylpyrrolidone was used to stabilize the nanorods in organic solvents before undergoing ligand exchange with mercaptoundecanoic acid over extended times (Supporting Information). When rod-shaped functionalized PMMA templates were exposed to a suspension of these nanorods, no assembly was observed at shorter times due to the lack of interaction between the amine groups and the thiol-capped nanorods. However, after $24 \mathrm{~h}$ and PMMA lift-off, assembled and partially aligned gold nanorods were observed as shown in Figure S5.

Most studies of assembled arrays employ gold nanoparticles due to their colloid stability, tunable optical properties and well-defined thiol chemistry. When DCA of silver nanoparticles was attempted, the filling efficiency was considerably lower than for gold nanoparticles, irrespective of ionic strength (Figure S6).

Transfer to Flexible Polymer Films. The key restriction for this application of DCA concerns the need for a hydroxylated substrate, which restricts the general applicability of this technique. However, as others have shown, it is possible to accurately and quantitatively transfer nanostructures from one substrate to another using polymer films as transfer stamps. ${ }^{54-55}$

We found that these arrays can be easily transferred by casting a PVA film over the array or by curing PDMS over the array, shown schematically in Figure 4a. In Figure 4b 
we see the topographical map obtained using AFM of the polymer film after encapsulating the arrays. A small fraction of the nanoparticles appear exposed, with only a $12 \%$ variation in the measured vertical height: $14.2 \pm 1.7 \mathrm{~nm}$. The dark field light microscopy images of the arrays on silicon, and embedded in the PVA films are shown in Figure 4c. Near quantitative transfer is achieved with a perfect mirror image of the array on silicon compared with the array in PVA.

(a)

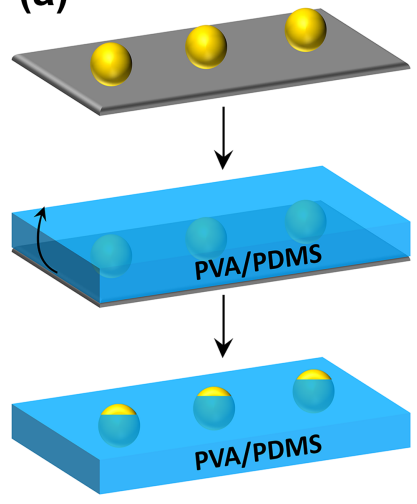

(b)
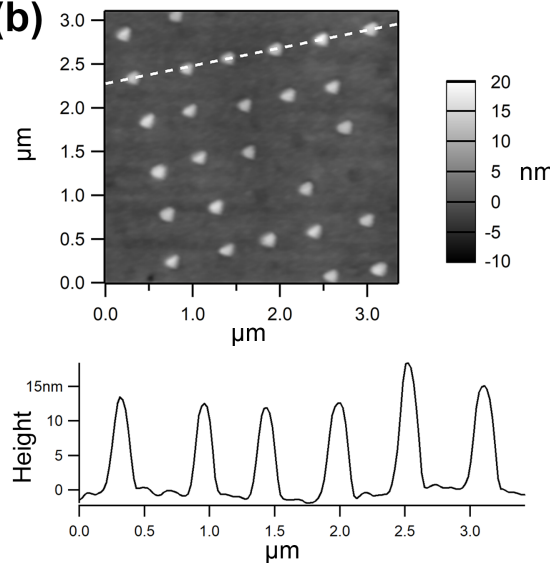

(c)

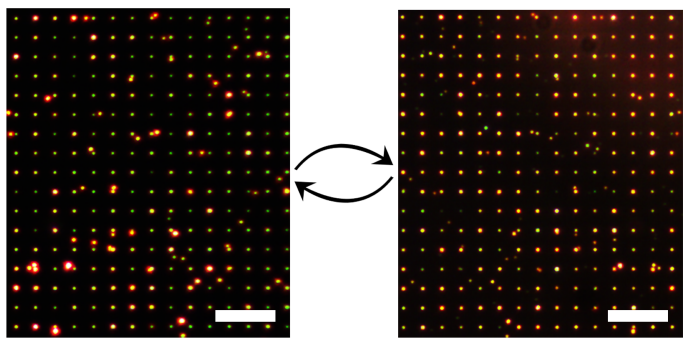

On Si

Figure 4. (a) Schematic of the embedding of nanoparticles in PVA or PDMS films. Lifting off a PVA film, via solvent casting, or a PDMS film, via curing, from the array generates an embedded plasmonic array. Note a small fraction ( $\mathrm{ca}$. $28 \%$ of the total nanoparticle surface area) is exposed. (b) Topographic image of an embedded array in PVA via AFM (above) and a topographic cross-section (below) showing the exposed nanoparticle surface. The gold nanoparticles were BSPP-capped, $72 \mathrm{~nm}$ in diameter, assembled on silicon at $78 \mathrm{pM}, \mathrm{pH} \mathrm{7.4}$, and $9.5 \mathrm{mM}$ ionic strength over $10 \mathrm{~min}$. (c) Dark field light microscopy image of an array of $72 \mathrm{~nm}$ gold nanoparticles on a silicon wafer (left) and the same array embedded in a $4 \mu \mathrm{m}$ thick PVA film (right). Scale bar, $12 \mu \mathrm{m}$.

\section{Discussion}

Through the approach described above, one can assemble either single or multiple gold nanoparticles into periodic arrays. While the amine groups drive the binding of the initial nanoparticle into the well, it is not a lack of attraction that prevents further gold particles from adsorbing, rather it is the double layer repulsion between adjacent nanoparticles. Indeed, we observe conditions where a single nanoparticle deposits in a well more than $10 x$ its size. Upon addition of salt, however, the electrostatic double layer around each 
nanoparticle is compressed meaning the gold nanoparticles can approach closer and pack more densely within each square well.

Interestingly, the nanoparticles are rarely in a close packed formation within each well, even after immersing the substrate for long times in the suspension, implying that the nanoparticle is relatively static upon adsorbing to the substrate. This leads to unusual kinetically-trapped cluster geometries, such as L-shapes and pyramids, which would be otherwise challenging to form in bulk solution (Figure 2).

These kinetically trapped states highlight the strength of the bond formed between the functional groups on the substrate and the surface of the nanoparticle. Regarding the gold-amine interaction, when the nanoparticles were capped with thiols there was very little assembly despite the electrostatic attraction between substrate and particle. Conversely, when sodium citrate was used as a ligand significant non-specific binding occurred due to the easy displacement by residual amine, phosphine, or thiol molecules adsorbed on the background, necessitating a PMMA template liftoff. Utilizing the optimized silane and ligand chemistries, along with 30 min deposition times, a 105x105 array of $72 \mathrm{~nm}$ gold nanoparticles could be generated in a parallel manner with almost no non-specific adsorption (Figure S4).

It is worth highlighting that successful assembly was consistently observed across experiments with other nanoparticle sizes and at different times compared with the data presented in Figures 1 and 3 . However, the fact that only three times (excluding $t=0$ ) were studied, as presented in Figure 3, means the accuracy of the extracted exponents may suffer some systematic error, despite the goodness of fit at large well separations. Therefore, we cannot decisively conclude that filling is diffusion-limited. From the data presented, it appears that this CDA method suffers from lower filling efficiencies of high density patterns, i.e. when the functionalized pattern comprises more than ca. $20 \%$ of the substrate or when the inter-well spacing is below $400 \mathrm{~nm}$. Upon increasing the wellspacing, however, highly efficient and rapid assembly was found.

Contrasting with the success of gold nanoparticle deposition, assembling silver nanoparticles was more challenging. We propose two reasons for this observation: Firstly, the amine-silver interaction is weaker, meaning the sticking probability of silver 
nanoparticles to an aminated surface is less than that for gold. Secondly, a thin silver oxide passivating layer would reduce the ligand density on the surface, thus reducing the attractive interactions between the positively charged surface and the anionic nanoparticles in suspension.

Despite the initial apparent non-generality of this approach, where hydroxylated substrates were needed, we found the arrays transferred easily via polymer lift-off techniques. These routes to embedded arrays of nanoparticles are of interest for reactions which are particularly surface dependent, such as plasmonic biosensors and catalysis, as well as mechanochromic plasmonic materials. Alternatively, the nanoparticle arrays can be embedded within the existing hydrophobic PMMA layer through heating the substrate to $140{ }^{\circ} \mathrm{C}$, above the glass transition temperature of the polymer (Figure S7). Rather than lifting the nanoparticle off the surface, the PMMA reflowed around the particle arrays, again demonstrating the strong particle-substrate bonds.

Conclusions: We have demonstrated a method for placing single nanoparticles onto surfaces with nanometre spatial resolution using EBL to generate nanowells, which can be chemically functionalised. This technique brings together the high-resolution localization of particles using a top-down fabrication approach with high quality singlecrystal nanoparticle synthesis. In principle, any arbitrary nanoparticle pattern can be produced, with the primary limitation being the resolution of the EBL writing beam. Assembly involves diffusion of the nanoparticles into the wells and binding to a molecular adhesive to prevent desorption. Double layer interactions play a key role and determine the ease of penetration into the wells. While the approach is complementary to other techniques, such as capillary force assembly and electrophoretic deposition, the rapid assembly method presented here is extremely efficient for nanoparticles in the range of $20-200+n m$ and can be scaled up using existing nanoimprint lithography tools.

Acknowledgements: The authors thank the ARC for Support through CE170100026 and LP110200319. This work was performed in part at the Melbourne Centre for 
Nanofabrication (MCN) in the Victorian Node of the Australian National Fabrication Facility (ANFF). We would like to thank Sergey Rubanov from the Bio21 Advanced Microscopy Facility (the University of Melbourne). CK acknowledges support from the Swiss National Science Foundation through an Early Postdoc.Mobility (P2FRP2_158730). 


\section{References}

1. Shipway, A. N.; Katz, E.; Willner, I., Nanoparticle arrays on surfaces for electronic, optical, and sensor applications. ChemPhysChem 2000, 1 (1), 18-52.

2. Terris, B. D.; Thomson, T., Nanofabricated and self-assembled magnetic structures as data storage media. Journal of Physics D-Applied Physics 2005, 38 (12), R199-R222.

3. Barrow, S. J.; Wei, X.; Baldauf, J. S.; Funston, A. M.; Mulvaney, P., The surface plasmon modes of self-assembled gold nanocrystals. Nat. Commun. 2012, 3, 1275.

4. Grzelczak, M.; Vermant, J.; Furst, E. M.; Liz-Marzan, L. M., Directed self-assembly of nanoparticles. ACS Nano 2010, 4 (7), 3591-605.

5. Redl, F. X.; Cho, K. S.; Murray, C. B.; O'Brien, S., Three-dimensional binary superlattices of magnetic nanocrystals and semiconductor quantum dots. Nature 2003, 423 (6943), 968-71.

6. Kooy, N.; Mohamed, K.; Pin, L. T.; Guan, O. S., A review of roll-to-roll nanoimprint lithography. Nanoscale Res. Lett. 2014, 9 (1), 320.

7. Halas, N. J.; Lal, S.; Chang, W. S.; Link, S.; Nordlander, P., Plasmons in Strongly Coupled Metallic Nanostructures. Chem. Rev. 2011, 111 (6), 3913-3961.

8. Hagglund, C.; Kasemo, B., Nanoparticle Plasmonics for 2D-Photovoltaics: Mechanisms, Optimization, and Limits. Opt. Express 2009, 17 (14), 11944-11957.

9. Bosman, M.; Zhang, L.; Duan, H.; Tan, S. F.; Nijhuis, C. A.; Qiu, C. W.; Yang, J. K., Encapsulated annealing: enhancing the plasmon quality factor in lithographically-defined nanostructures. Sci. Rep. 2014, 4, 5537.

10. Laroche, T.; Vial, A.; Roussey, M., Crystalline structure's influence on the near-field optical properties of single plasmonic nanowires. Appl. Phys. Lett. 2007, 91 (12).

11. Cerf, A.; Tian, H. C.; Craighead, H. G., Ordered arrays of native chromatin molecules for high-resolution imaging and analysis. ACS Nano 2012, 6 (9), 7928-34.

12. Dai, Q.; Frommer, J.; Berman, D.; Virwani, K.; Davis, B.; Cheng, J. Y.; Nelson, A., High-throughput directed self-assembly of core-shell ferrimagnetic nanoparticle arrays. Langmuir 2013, 29 (24), 7472-7472.

13. Hamon, C.; Novikov, S.; Scarabelli, L.; Basabe-Desmonts, L.; Liz-Marzán, L. M., Hierarchical Self-Assembly of Gold Nanoparticles into Patterned Plasmonic Nanostructures. ACS Nano 2014, 8 (10), 10694-10703.

14. Mehraeen, S.; Asbahi, M.; Fuke, W.; Yang, J. K.; Cao, J.; Tan, M. C., Directed SelfAssembly of sub-10 nm Particles: Role of Driving Forces and Template Geometry in Packing and Ordering. Langmuir 2015, 31 (31), 8548-8557.

15. Ni, S.; Wolf, H.; Isa, L., Programmable Assembly of Hybrid Nanoclusters. Langmuir 2018, 34 (7), 2481-2488.

16. Malaquin, L.; Kraus, T.; Schmid, H.; Delamarche, E.; Wolf, H., Controlled particle placement through convective and capillary assembly. Langmuir 2007, 23 (23), 11513-11521.

17. Xia, Y. N.; Yin, Y. D.; Lu, Y.; McLellan, J., Template-assisted self-assembly of spherical colloids into complex and controllable structures. Adv. Funct. Mater. 2003, 13 (12), 907-918.

18. Cui, Y.; Björk, M. T.; Liddle, J. A.; Sönnichsen, C.; Boussert, B.; Alivisatos, A. P., Integration of Colloidal Nanocrystals into Lithographically Patterned Devices. Nano Lett. 2004, 4 (6), 1093-1098. 
19. Flauraud, V.; Mastrangeli, M.; Bernasconi, G. D.; Butet, J.; Alexander, D. T. L.; Shahrabi, E.; Martin, O. J. F.; Brugger, J., Nanoscale topographical control of capillary assembly of nanoparticles. Nat. Nanotech. 2016, 12, 73.

20. Pinedo Rivera, T.; Lecarme, O.; Hartmann, J.; Rossitto, E.; Berton, K.; Peyrade, D., Assisted convective-capillary force assembly of gold colloids in a microfluidic cell: Plasmonic properties of deterministic nanostructures. Journal of Vacuum Science \& Technology B 2008, 26 (6), 2513-2519.

21. Gargiulo, J.; Violi, I. L.; Cerrota, S.; Chvatal, L.; Cortes, E.; Perassi, E. M.; Diaz, F.; Zemanek, P.; Stefani, F. D., Accuracy and Mechanistic Details of Optical Printing of Single Au and Ag Nanoparticles. ACS Nano 2017, 11 (10), 9678-9688.

22. Urban, A. S.; Lutich, A. A.; Stefani, F. D.; Feldmann, J., Laser printing single gold nanoparticles. Nano Lett. 2010, 10 (12), 4794-4798.

23. Moutet, P.; Lacroix, L.-M.; Robert, A.; Impéror-Clerc, M.; Viau, G.; Ressier, L., Directed Assembly of Single Colloidal Gold Nanowires by AFM Nanoxerography. Langmuir 2015, 31 (14), 4106-4112.

24. Barry, C. R.; Gu, J.; Jacobs, H. O., Charging process and coulomb-force-directed printing of nanoparticles with sub-100-nm lateral resolution. Nano Lett. 2005, 5 (10), 2078-2084.

25. Chad, R. B.; Michael, G. S.; Nyein, Z. L.; Heiko, O. J., Printing nanoparticles from the liquid and gas phases using nanoxerography. Nanotechnology 2003, 14 (10), 1057-1063.

26. Moutet, P.; Deram, P.; Sangeetha, N. M.; Ressier, L., Dynamics of DielectrophoreticForce-Directed Assembly of NaYF4 Colloidal Nanocrystals into Tunable Multilayered Micropatterns. J. Phys. Chem. Lett. 2014, 5 (17), 2988-2993.

27. Diaz, R.; Palleau, E.; Poirot, D.; Sangeetha, N. M.; Ressier, L., High-throughput fabrication of anti-counterfeiting colloid-based photoluminescent microtags using electrical nanoimprint lithography. Nanotechnology 2014, 25 (34), 345302.

28. Wei, Z.; Barlow, D. E.; Sheehan, P. E., The Assembly of Single-Layer Graphene Oxide and Graphene Using Molecular Templates. Nano Lett. 2008, 8 (10), 3141-3145.

29. Wang, Y.; Maspoch, D.; Zou, S.; Schatz, G. C.; Smalley, R. E.; Mirkin, C. A., Controlling the shape, orientation, and linkage of carbon nanotube features with nano affinity templates. Proc. Natl. Acad. Sci. U. S. A. 2006, 103 (7), 2026-2031.

30. Rao, S. G.; Huang, L.; Setyawan, W.; Hong, S., Large-scale assembly of carbon nanotubes. Nature 2003, 425, 36-37.

31. Zou, S.; Maspoch, D.; Wang; Mirkin, C. A.; Schatz, G. C., Rings of Single-Walled Carbon Nanotubes: Molecular-Template Directed Assembly and Monte Carlo Modeling. Nano Lett. 2007, 7 (2), 276-280.

32. Fan, Z.; Ho, J. C.; Jacobson, Z. A.; Yerushalmi, R.; Alley, R. L.; Razavi, H.; Javey, A., Wafer-Scale Assembly of Highly Ordered Semiconductor Nanowire Arrays by Contact Printing. Nano Lett. 2008, 8 (1), 20-25.

33. Ma, L.-C.; Subramanian, R.; Huang, H.-W.; Ray, V.; Kim, C.-U.; Koh, S. J., Electrostatic Funneling for Precise Nanoparticle Placement: A Route to Wafer-Scale Integration. Nano Lett. 2007, 7 (2), 439-445.

34. Zhou, X.; Xu, H.; Cheng, J.; Zhao, N.; Chen, S.-C., Flexure-based Roll-to-roll Platform: A Practical Solution for Realizing Large-area Microcontact Printing. Sci. Rep. 2015, 5, 10402.

35. Sabik, S.; Riet, J. d.; Yakimets, I.; Smits, E. In High resolution patterning for flexible electronics via roll-to-roll nanoimprint lithography, SPIE Advanced Lithography, SPIE: 2014; $\mathrm{p}$ 9. 
36. Gannon, G.; Larsson, J. A.; Greer, J. C.; Thompson, D., Quantification of Ink Diffusion in Microcontact Printing with Self-Assembled Monolayers. Langmuir 2009, 25 (1), 242-247.

37. Lin, Y.; Daga, V. K.; Anderson, E. R.; Gido, S. P.; Watkins, J. J., Nanoparticle-Driven Assembly of Block Copolymers: A Simple Route to Ordered Hybrid Materials. J. Am. Chem. Soc. 2011, 133 (17), 6513-6516.

38. Jang, S. G.; Kramer, E. J.; Hawker, C. J., Controlled Supramolecular Assembly of Micelle-Like Gold Nanoparticles in PS-b-P2VP Diblock Copolymers via Hydrogen Bonding. $J$. Am. Chem. Soc. 2011, 133 (42), 16986-16996.

39. Son, J. G.; Bae, W. K.; Kang, H.; Nealey, P. F.; Char, K., Placement Control of Nanomaterial Arrays on the Surface-Reconstructed Block Copolymer Thin Films. ACS Nano 2009, 3 (12), 3927-3934.

40. Nepal, D.; Onses, M. S.; Park, K.; Jespersen, M.; Thode, C. J.; Nealey, P. F.; Vaia, R. A., Control over Position, Orientation, and Spacing of Arrays of Gold Nanorods Using Chemically Nanopatterned Surfaces and Tailored Particle-Particle-Surface Interactions. ACS Nano 2012, 6 (6), 5693-5701.

41. Gilles, S.; Kaulen, C.; Pabst, M.; Simon, U.; Offenhäusser, A.; Mayer, D., Patterned selfassembly of gold nanoparticles on chemical templates fabricated by soft UV nanoimprint lithography. Nanotechnology 2011, 22 (29), 295301.

42. Yang, L. L.; Yan, B.; Premasiri, W. R.; Ziegler, L. D.; Dal Negro, L.; Reinhard, B. M., Engineering Nanoparticle Cluster Arrays for Bacterial Biosensing: The Role of the Building Block in Multiscale SERS Substrates. Adv. Funct. Mater. 2010, 20 (16), 2619-2628.

43. Jiang, L.; Sun, Y.; Nowak, C.; Kibrom, A.; Zou, C.; Ma, J.; Fuchs, H.; Li, S.; Chi, L.; Chen, X., Patterning of plasmonic nanoparticles into multiplexed one-dimensional arrays based on spatially modulated electrostatic potential. ACS Nano 2011, 5 (10), 8288-8294.

44. Jiang, L.; Chen, X.; Lu, N.; Chi, L., Spatially confined assembly of nanoparticles. Acc. Chem. Res. 2014, 47 (10), 3009-3017.

45. Chou, S. Y.; Krauss, P. R.; Renstrom, P. J., Nanoimprint lithography. Journal of Vacuum Science \& Technology B: Microelectronics and Nanometer Structures Processing, Measurement, and Phenomena 1996, 14 (6), 4129-4133.

46. Funston, A. M.; Novo, C.; Davis, T. J.; Mulvaney, P., Plasmon Coupling of Gold Nanorods at Short Distances and in Different Geometries. Nano Lett. 2009, 9 (4), 1651-1658.

47. Sardar, R.; Funston, A. M.; Mulvaney, P.; Murray, R. W., Gold Nanoparticles: Past, Present, and Future. Langmuir 2009, 25 (24), 13840-13851.

48. Bastus, N. G.; Comenge, J.; Puntes, V., Kinetically Controlled Seeded Growth Synthesis of Citrate-Stabilized Gold Nanoparticles of up to $200 \mathrm{~nm}$ : Size Focusing versus Ostwald Ripening. Langmuir 2011, 27 (17), 11098-11105.

49. Ye, X.; Zheng, C.; Chen, J.; Gao, Y.; Murray, C. B., Using binary surfactant mixtures to simultaneously improve the dimensional tunability and monodispersity in the seeded growth of gold nanorods. Nano Lett. 2013, 13 (2), 765-771.

50. Bastus, N. G.; Merkoci, F.; Piella, J.; Puntes, V., Synthesis of Highly Monodisperse Citrate-Stabilized Silver Nanoparticles of up to $200 \mathrm{~nm}$ : Kinetic Control and Catalytic Properties. Chem. Mater. 2014, 26 (9), 2836-2846.

51. Jiang, L.; Wang, W.; Fuchs, H.; Chi, L., One-dimensional arrangement of gold nanoparticles with tunable interparticle distance. Small 2009, 5 (24), 2819-2822. 
52. Kinnear, C.; Burnand, D.; Clift, M. J.; Kilbinger, A. F.; Rothen-Rutishauser, B.; PetriFink, A., Polyvinyl alcohol as a biocompatible alternative for the passivation of gold nanorods. Angew. Chem. Int. Ed. 2014, 53 (46), 12613-12617.

53. Kinnear, C.; Dietsch, H.; Clift, M. J.; Endes, C.; Rothen-Rutishauser, B.; Petri-Fink, A., Gold nanorods: controlling their surface chemistry and complete detoxification by a two-step place exchange. Angew. Chem. Int. Ed. 2013, 52 (7), 1934-1938.

54. Li, H.; Wu, J. M. T.; Huang, X.; Yin, Z. Y.; Liu, J. Q.; Zhang, H., A Universal, Rapid Method for Clean Transfer of Nanostructures onto Various Substrates. ACS Nano 2014, 8 (7), 6563-6570.

55. Tebbe, M.; Mayer, M.; Glatz, B. A.; Hanske, C.; Probst, P. T.; Muller, M. B.; Karg, M.; Chanana, M.; Konig, T. A. F.; Kuttner, C.; Fery, A., Optically anisotropic substrates via wrinkleassisted convective assembly of gold nanorods on macroscopic areas. Faraday Discuss. 2015, 181, 243-260. 
For Table of Contents Only
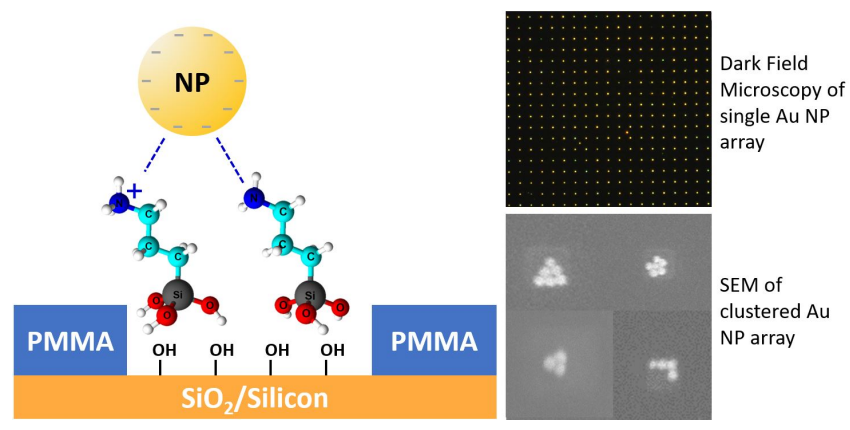


\section{University Library}

\section{- M M N E R VA A gateway to Melbourne's research publications}

Minerva Access is the Institutional Repository of The University of Melbourne

Author/s:

Kinnear, C;Cadusch, J;Zhang, H;Lu, J;James, TD;Roberts, A;Mulvaney, P

Title:

Directed Chemical Assembly of Single and Clustered Nanoparticles with Silanized Templates

Date:

2018-06-26

Citation:

Kinnear, C., Cadusch, J., Zhang, H., Lu, J., James, T. D., Roberts, A. \& Mulvaney, P. (2018). Directed Chemical Assembly of Single and Clustered Nanoparticles with Silanized Templates. LANGMUIR, 34 (25), pp.7355-7363. https://doi.org/10.1021/ acs.langmuir.8b00775.

Persistent Link:

http://hdl.handle.net/11343/284279 